\title{
EPIDERMOLISIS POR CHIKUNGUNYA POR TRANSMISIÓN AUTÓCTONA EN REPÚBLICA DOMINICANA. CASO ATÍPICO Y SEVERO EN UN RECIÉN NACIDO (RN)
}

\author{
Epidermolysis by Chikungunya by native transmission in the \\ Dominican Republic. Atypical and severe case in a Newborn (RN)
}

\section{Virgen Gómez-Alba, Maylen Chalas y Eduardo Michelen}

Recibido: 26 de octubre, 2019 • 6 de diciembre, 2019

Cómo citar: Gómez Alba V, Chalas M, Michelen E. Epidermolisis por chikungunya por transmisión autóctona en República Dominicana. Caso atípico y severo en un recién nacido (RN). cysa [Internet]. 3 de marzo de 2020 [citado 4 de marzo de 2020];4(1):71-8. Disponible en: https://revistas.intec.edu.do/index.php/cisa/article/view/1664

Resumen

Introducción: chikunguya, enfermedad transmitida por la picadura del mosquito del género Aedes, suele ser asintomática y benigna en su evolución. Sin embargo, se han observado manifestaciones clínicas atípicas y severas en niños durante la infección por chikungunya.

Objetivo: presentar caso epidermólisis por chikungunya en $\mathrm{RN}$ de 24 días de edad durante la epidemia del 2014 en República Dominicana.

Caso clínico: $\mathrm{RN}$ de 27 días de edad con lesión inicial ampollosa en tórax que luego se generalizó correspondiendo a una epidermólisis. Se observó una buena evolución y lesiones residuales híper e hipocrómicas en piel.

Palabras clave: chikungunya; epidermolisis; recién nacido; transmisión autóctona; República Dominicana.

\footnotetext{
a Pediatra Infectóloga Centro Médico Universidad Central del Este (CM-UCE), República Dominicana

Correo-e: virgen.gomez@yahoo.es
}

\begin{abstract}
Introduction: Chikungunya, an infectious disease transmitted by the bite of an Aedes mosquito, tends to be an asymptomatic and benign course. However, atypical and severe clinical manifestations have been observed in children during Chikungunya infection.

Objective: To present a case of epidermolysis by Chikungunya in a 24-day-old newborn during the 2014 epidemic in the Dominican Republic.

Case report: Case of 24-day-old newborn presenting with an initial bullous thorax lesion that later spread diffusely developing into an epidermolysis. After management, good evolution was observed. However, residual hyper and hypochromic skin lesions remained.
\end{abstract}

Keywords: Chikungunya; epidermolysis; newborn; native transmission; Dominican Republic.

\footnotetext{
b Pediatra Centro Médico Universidad Central del Este (CM-UCE), República Dominicana Correo-e: maylenchalas@hotmail.com

${ }^{\mathrm{c}}$ Estudiante de Medicina Universidad de Puerto Rico (UPI), Puerto Rico Correo-e: michedu3@hotmail.com
} 


\section{Introducción}

El virus de chikungunya (CHIKV), es una arbovirosis emergente, transmitida por la picadura de mosquitos del género Aedes y descrita por primera vez en Tanzania en el 1952. En el $80 \%$ de los casos puede ser asintomática y afecta a millones de personas en el mundo. ${ }^{1,2}$

Es endémica en el sudeste de Asia, África y Oceanía y en diciembre del 2013 se confirman los primeros casos autóctonos en la región de las Américas, ocasionando epidemias importantes en diferentes países, siendo República Dominicana (RD) el país que mayor número de casos ha reportado durante la epidemia. ${ }^{1,2}$

El 6 de diciembre del 2013 la Organización Panamericana de la Salud (OPS) notifica la confirmación de dos casos de transmisión autóctona del virus de chikungunya en la isla de Saint Martin; casos importados se habían registrado en Estados Unidos, Canadá, Guyana Francesa, Martinica, Guadalupe y Brasil. El 9 de diciembre de 2013 la OPS emitió por primera vez la alerta regional sobre la transmisión autóctona del virus chikungunya en la Región de las Américas. ${ }^{1,2}$

En República Dominicana, los primeros casos se reportan la semana epidemiológica 16, actualizada al 18 de abril del 2014, aportando el mayor número de casos en nuestro continente, 539,099 casos $48 \%$ de todos los casos reportados en las Américas para la semana 52 del 2014, con solo 84 casos confirmados y 6 muertes asociadas. ${ }^{3,4}$

Aunque es una enfermedad relativamente benigna, pues la gran mayoría de los pacientes se recuperan en los primeros cinco días, pueden ocurrir complicaciones afectando cualquier órgano o sistema: convulsiones febriles, meningoencefalitis, Síndrome Guillian-Barre, diplopía, afasia, miocarditis, descompensación cardiovascular, hepatitis fulminante, pancreatitis, daño renal, insuficiencia respiratoria, hemorragias menores, erupción vesículo-bullosa, epidermolisis, entre otras. El $20 \%$ de los pacientes con afectación neurológica pueden presentar secuelas si ocurre en edades tempranas con manifestaciones iniciales graves. ${ }^{3,5-14}$

La afectación de la piel ocurre en el 40-50\% de los casos. Entre el tercer y quinto días del inicio de la enfermedad aparece rash maculopapular, que palidece o desaparece a la vitropresión, usualmente afecta tórax y extremidades y, en menor proporción, la cara. Afecta también palmas de las manos y plantas de los pies, y puede ser pruriginoso o edematoso. Con menor frecuencia aparecen lesiones exfoliativas que descaman y lesiones vesículo ampollosas, principalmente en recién nacidos. ${ }^{15}$

Reportamos un caso de epidermólisis en un RN de 24 días de edad, en el contexto de la epidemia de chikungunya en $\mathrm{RD}$, con historia de que sus padres $y$ hermanos presentaron enfermedad febril aguda acompañada de erupción, dolor articular, cefalea y ojos rojos, lo consideramos como enfermedad atípica y severa por chikungunya, que se resolvió sin secuelas, lo que motiva nuestra presentación.

\section{Caso clínico}

RN masculino de 24 días de edad, con historia de fiebre de inicio brusco $\left(39.5^{\circ} \mathrm{C}\right)$, llanto frecuente, irritabilidad y erupción maculo papular diseminada en piel, fue admitido en un centro médico de su comunidad con diagnóstico de Chikungunya.

Al tercer día de inicio de la enfermedad, presenta lesión ampollar en región anterior del tórax, por lo que es referido a nuestro centro, Centro Médico Universidad Central del Este (CM-UCE).

Producto de embarazo a término con chequeos prenatales adecuados, parto por cesárea, con pará- 
metros antropométricos normales, clasificado como $\mathrm{RN}$ a término adecuado para edad gestacional.

En los antecedentes se reporta que sus padres y sus dos hermanos presentaron cuadro febril con erupción, ojos rojos y dolor en las articulaciones de las manos y pies, diagnosticados como enfermedad por virus de chikungunya.

En la evaluación clínica inicial encontramos un recién nacido a término, eutrófico, irritable, afebril, hidratado, con erupción máculo-papular generalizada y lesión ampollosa denudada en tórax (figuras 1a y 1b) y ampollas en miembros inferiores, por lo que es ingresado con diagnóstico de epidermólisis por virus chikungunya. D/C Estafilococia/ Estreptococia.

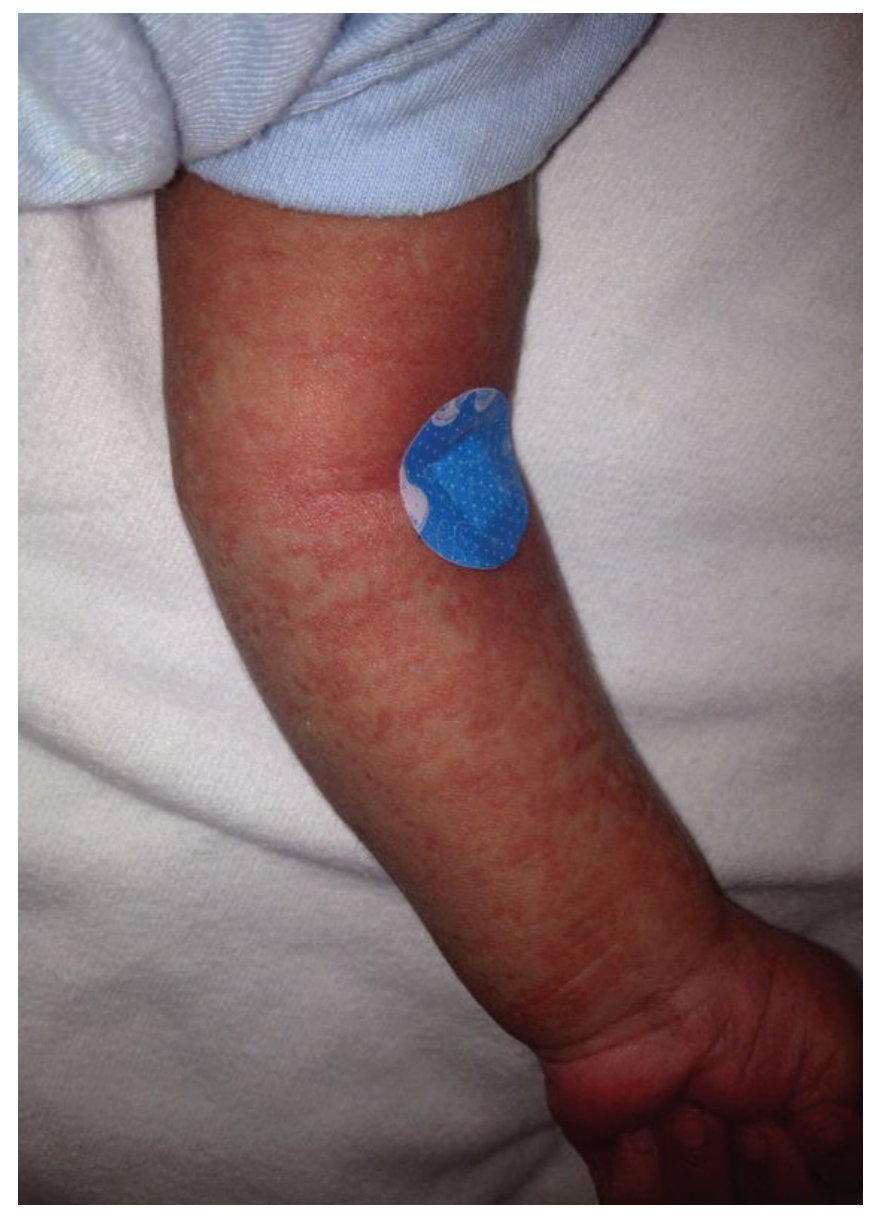

Figura 1a. Erupción maculo-papular

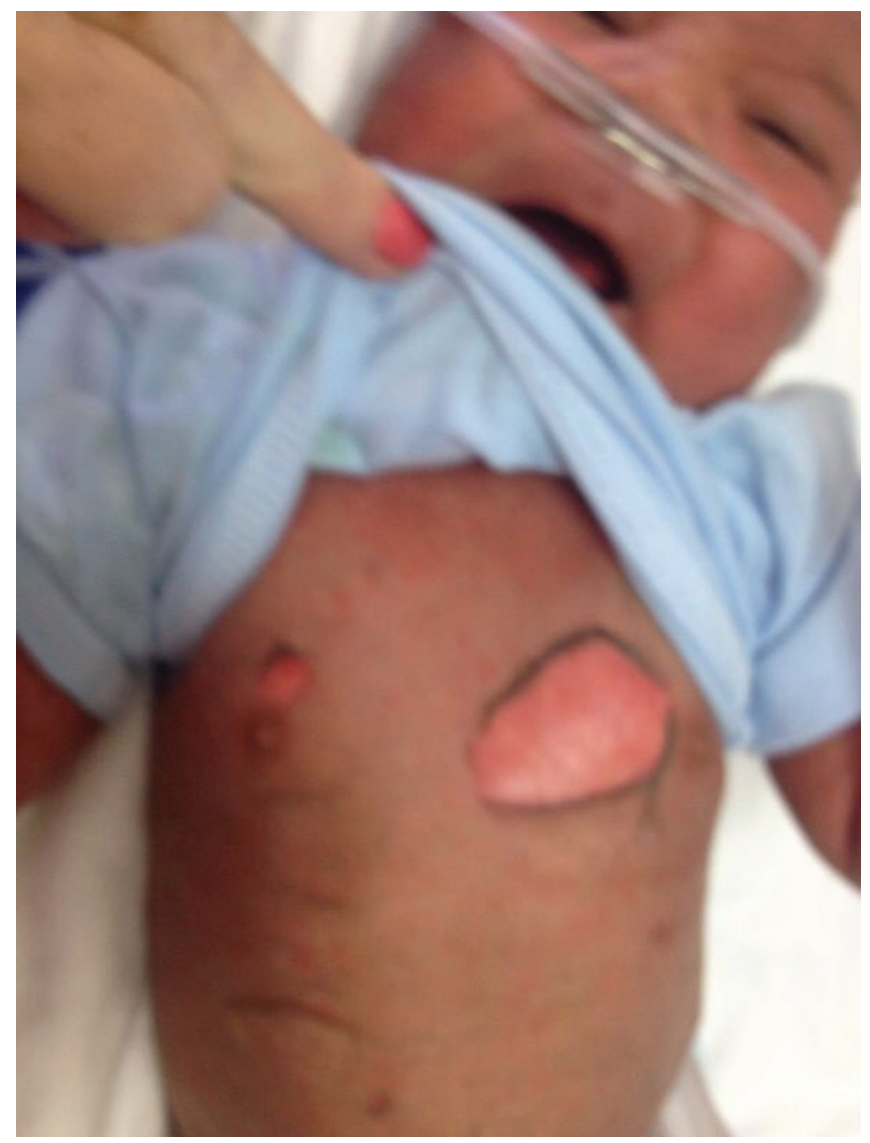

Figura 1b. Lesiones ampollosas en tronco

Fuente: Departamento de Pediatría Centro Médico UCE

Luego de la toma de muestras para hemograma, ERS, PCR cuantitativa, electrolitos, orina, hemocultivo, cultivo de lesiones de la piel, se inicia manejo con soluciones EV, vancomicina y amikacina, apego estricto a asepsia y antisepsia, uso de ropa y sábanas estériles.

Al segundo día de su ingreso, quinto día de su enfermedad, presenta dificultad respiratoria, se realiza radiografía de tórax, en la que no se evidencian alteraciones, por lo que se inicia CPAP con buena respuesta.

Las lesiones ampollosas se diseminaron por todo el cuerpo haciéndose confluentes, con áreas denudadas limpias e hiperpigmentación en cara. (Figuras de $2 \mathrm{a}$ $2 \mathrm{~b}, 2 \mathrm{c}, 2 \mathrm{~d})$ 

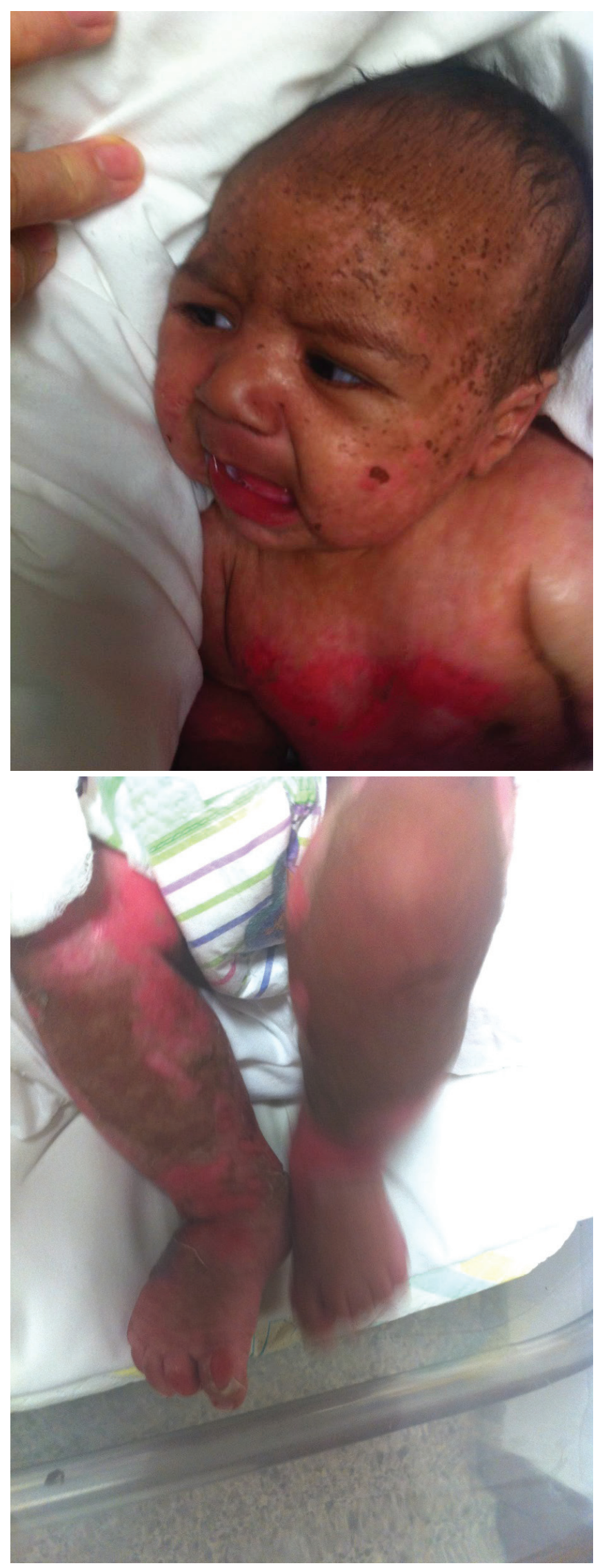
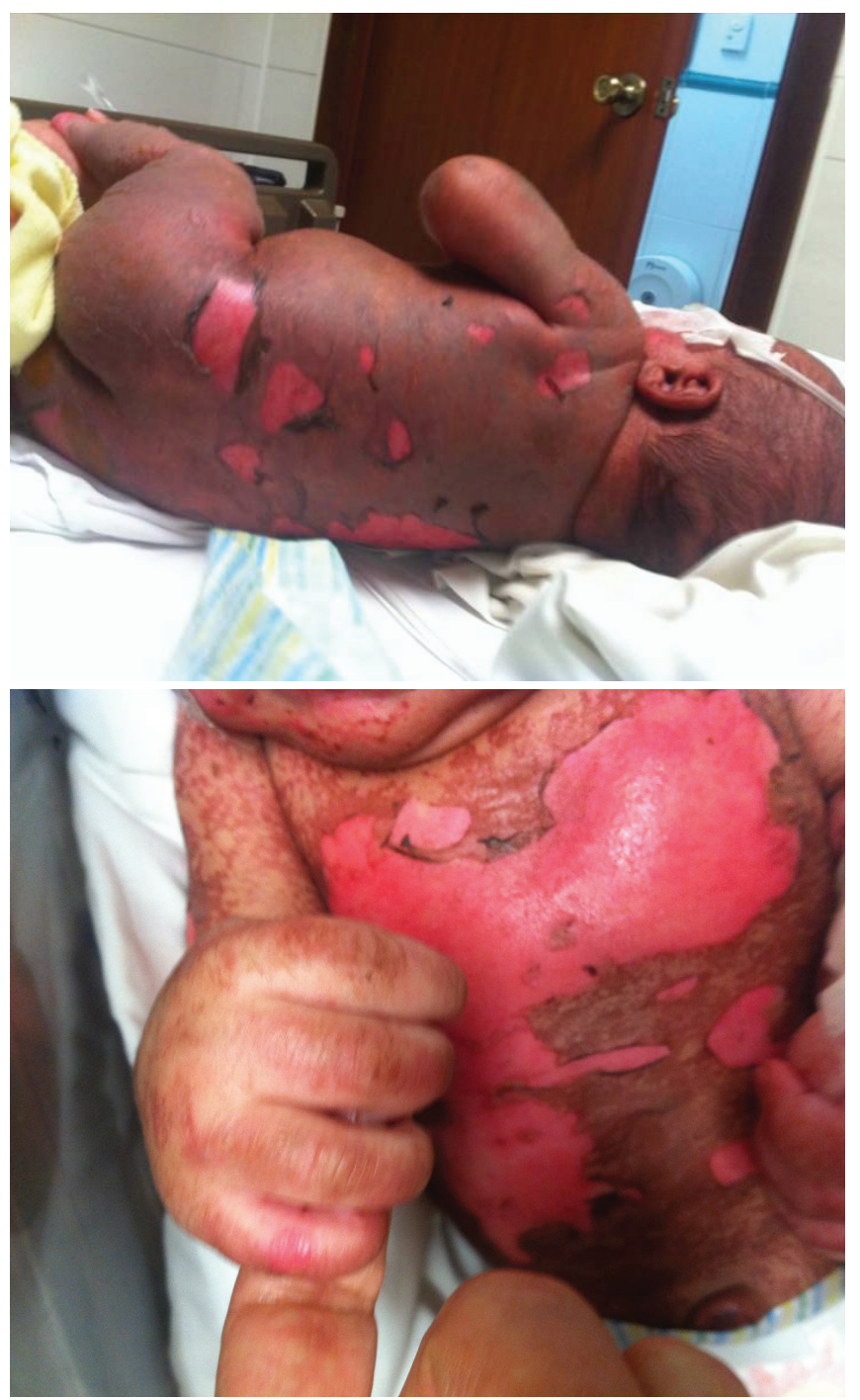

Figuras 2a, 2b,2c, 2d. Pigmentación en cara y lesiones ampollosas denudadas en tronco y miembros inferiores

Fuente: Departamento de Pediatría Centro Médico UCE

El hemograma inicial muestra anemia, leucopenia y trombocitopenia leves con linfopenia moderada; electrolitos y examen de orina normales, hemocultivo y cultivo, lesiones de piel, no crecimiento de microorganismos.

Permanece ingresado por 10 días con buena evolución clínica, se egresa en buen estado general, lesiones piel con resolución parcial de lesiones ampollosas y áreas de hipo e hiperpigmentación. (Figuras 3a, 3b,3c). 

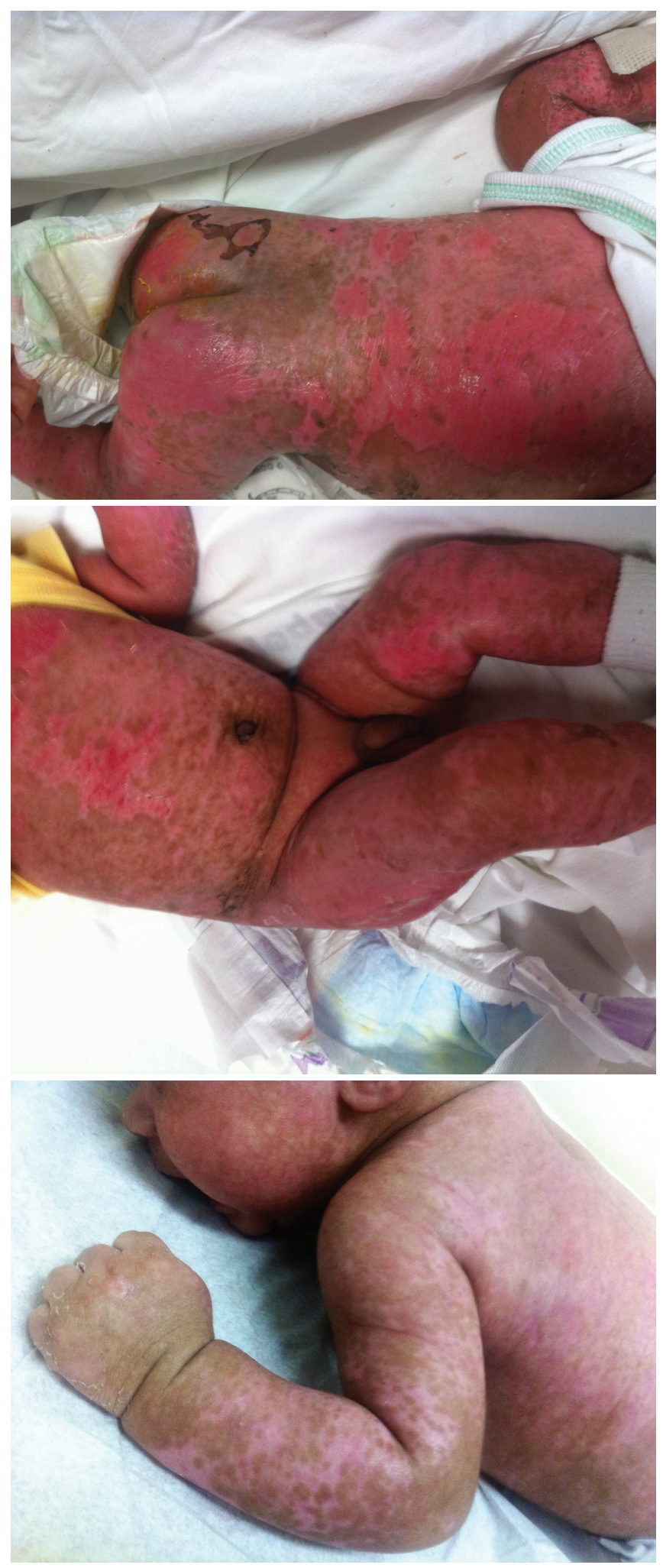

Figuras 3a,3b,3c. Evolución de lesiones ampollosas, manchas hipo e hipercrómicas residuales

Fuente: Departamento de Pediatría Centro Médico UCE
En su seguimiento ambulatorio a las dos semanas del egreso, se confirma resolución de lesiones dejando manchas híper e hipo crómicas diseminadas por toda la piel, se indican filtro solar para su protección, vitaminas con hierro por anemia y medidas generales.

En su visita de los seis meses de edad, buen desarrollo psicomotor, persiste hipo e híper pigmentación de piel.

\section{Discusión}

Desde el 1952 cuando se aisló el virus de chikungunya, múltiples brotes y epidemias han ocurrido en el mundo, África, Asia, India, entre otros. En diciembre del 2013 las Américas entran dentro de los países afectados, cuando se confirman los dos primeros casos de transmisión autóctona en San Martin; en nuestro país, los primeros casos se reportan en abril del 2014, aportando la mayoría de casos de nuestro continente. ${ }^{1,2,5}$

Aunque la mayoría de los casos son asintomáticos y se resuelven sin complicaciones, se han descrito formas atípicas y severas que incluyen afectación neurológica, ocular, cardiovascular, renal, respiratorias, discrasias sanguíneas, hepatitis, pancreatitis, síndrome de secreción inadecuada de hormona antidiurética e hipoadrenalismo, entre otras. A nivel de piel puede presentarse hiperpigmentación fotosensible, úlceras, dermatosis vesículo-bullosas como nuestro caso, que presentó epidermólisis con lesiones ampollosas extensas, diseminadas, respetando solo la cara, donde se observó hiperpigmentación. ${ }^{3-5,11-16}$

Casos atípicos y severos han sido reportados en brotes y epidemias en diferentes países. En la epidemia del 2005-2006 ocurrida en la isla Reunión, la incidencia de infecciones atípicas y severas fueron reportadas en el 0,3\% de los casos, en ocasiones asociados al uso de AINES y ASA. Algunos pacientes 
presentaron insuficiencia pre-renal o exacerbación de algún daño renal preexistente. ${ }^{7-9}$

Los casos atípicos y severos son más frecuentes en niños que en adultos, siendo los $\mathrm{RN}$ y los menores de 2 años los más afectados. Cambios pigmentarios y lesiones bullosas extensas en piel se presentan, principalmente, en menores de 6 meses, que pueden cubrir hasta el $35 \%$ de la superficie de la piel, el $10 \%$ puede presentar hemorragias. ${ }^{15,18}$

Las manifestaciones atípicas y severas pueden estar ligadas a cambios en el genoma del virus, como se demostró en la India con la mutación K221E en los genotipos del linaje Centro-Este de Sudáfrica (ECSA) y CHIKV-WH, identificados en cuadros cutáneos severos en $\mathrm{RN}$ e infantes graves. ${ }^{19}$

El envolvimiento de las extremidades inferiores puede desarrollarse 2 a 3 días después de iniciada la fiebre, reportándose lesiones vesiculares simétricas y acrocianosis sin alteraciones hemodinámicas. Las vesículas y bullas pueden denudarse evolucionando a epidermólisis, sin participación de las mucosas, causando necrosis focal de la epidermis con infiltración leucocitaria, como en nuestro caso, que inicia con una lesión ampollosa en tórax, apareciendo lesiones ampollosas de dimensiones diferentes y confluentes, que luego se diseminaron en todo el cuerpo, respondiendo a su manejo y dejando solo manchas hipo e hipercrómicas como secuelas en piel. $6,8,11,13,17,20,21$

La infección perinatal también ha sido descrita en cerca del $50 \%$ de los $\mathrm{RN}$ de madres que tuvieron la enfermedad 4 días previos a 2 días post parto. La mayoría presenta manifestaciones clínicas al cuarto día de edad, con un rango de 3-7 días. Los casos que aparecen después del séptimo al noveno día se consideran de transmisión autóctona. ${ }^{7,14}$

Aunque no existe un tratamiento específico para el manejo de la enfermedad por CHIKV, y más aún para los casos severos, solo tratamiento sintomático, en nuestro caso el uso de antibioterapia para cubrir Staphilococcus aureus y Streptococcus pyogenes se justifica por la gran afectación de piel en un RN.

El uso de inmunoglobulina endovenosa se ha propuesto como una alternativa en el manejo de pacientes con enfermedad atípica y severa de piel, ya que se ha utilizado en pacientes con Síndrome de Guillain-Barré asociados al CHIKV, con buenos resultados. ${ }^{22}$

Nuestro caso correspondió a transmisión autóctona, ya que en el RN se considera transmisión vertical cuando el cuadro clínico inicia entre el tercer y séptimo día de edad, con un promedio de 4 días. De igual modo, porque la enfermedad se presentó a los 24 días de nacido, desarrollando epidermólisis severa, con distress respiratorio y una respuesta adecuada al manejo, cuya evolución fue satisfactoria y solo presentó manchas hipo e híper crómicas secundarias a las lesiones ampollosas generalizadas durante el período agudo de la enfermedad, en la etapa de recuperación y curación.

\section{Conclusión}

CHIKV, aunque usualmente produce enfermedad asintomática y leve, puede presentar manifestaciones atípicas y severas en piel, más frecuente en recién nacidos y menores de 2 años que ponen en peligro sus vidas, por lo que un manejo temprano, intensivo y oportuno es prioritario para salvar sus vidas y evitar complicaciones y secuelas.

\section{Recomendaciones}

El conocimiento de la evolución de la enfermedad por CHIKV en las últimas epidemias, de un cuadro leve a presentaciones atípicas y severas como nuestro caso, nos comprometen a investigar cambios en los genotipos virales y alternativas en el tratamiento de estos casos. 


\section{Bibliografía}

1. Organización Panamericana de la Salud. Alerta epidemiológica. Fiebre por chikungunya. [Online]. Disponible en: http://www.paho.org/hq/index. php?o p t i o s $_{\text {c o }} \mathrm{m}_{-} \mathrm{d}$ o c m a n $\& \mathrm{t}$ a s $\mathrm{k}=$ d o c_view\&gid=23807\&Itemid [Accessed 9 de diciembre de 2013].

2. Preparación y respuesta ante la eventual introducción del virus chikungunya en la Américas. Whashington DC. OPS 2011.SBN 978-9275-31632-0 Disponible en: http://www.paho. org/hq/index.php?option=com_docman \&task=doc_download\&gid=16985\&Itemid

3. Instrumento Para el Diagnóstico y Atención a Pacientes con sopecha de Arbovirosis. Washington D.C.: OPS; 2016. ISBN: 978-92-75-31936-9. Organización Pana

4. Moya J, Pimentel R, Puello J. Chikungunya: un reto para los servicios de salud de la República Dominicana. Rev Panam Salud Pública. 2014; 36(5): 331-5.

5. Número de casos reportados de chikungunya en países y territorios de las Américas por semanas. Casos acumulados a la Semana Epidemiológica 52 Actualizado al 29 de diciembre 2014.OPS/ OMS. Disponible en: https:www.paho.org/hq/ dmdocuments/2014/SE52/29-12-2014

6. Sebastian, M.R., Lodha, R. \& Kabra,. Chikungunya Infection in Children. Indian J Pediatr (2009) 76: 185. https://doi.org/10.1007/s12098009-0049-6

7. Bhat RM, Ramesh A, Nandakishore B, Sukumar D, Martis J, Kamath GH. Mucocutaneous manifestations of Chikungunya fever: a estudy from an epidemic in coastal Karnataka. Indian J Dermatol. 2011;56: 290-4.
8. Bandyopadhyay D, Ghosh SK. Mucocutaneous manifestations of Chikungunya fever. Indian J Dermatol. 2010;55: 64-7

9. Ramful D, Carbonnier M, Pasquet M, Bouhmani B, Ghazouani J, Noormahomed T, Beullier G, Attali T, Samperiz S, Fourmaintraux A, Alessandre JL. Mother-to-Child Transmission of Chikungunya Virus Infection. The Pediatric Infect Dis J. 2007 sep; 26(9): 811-5.

10. Muñoz C, Castillo J, Salas D, Valderrama M, Rangel C, Vargas H, Silva D. Manifestaciones mucocutáneas atípicas por chikungunya. Biomédica 2016;36:368-77. doi: http://dx.doi. org/10.7705/biomedica.v36i3.2760

11. Nkoghe D, Kassa RF, Caron M, Grard G, Mombo I, Bikie B, Paupy C, Becquart P, Bivigou U, Leroy E. Clinical Forms of Chikungunya in Gabon, 2010. PLoS Negl Trop Dis. 2012;6(2): e1517. doi:10.1371/journal.pntd.0001517

12. Gerardin P, Barau G, Michault A, Bintner M, Randrianaivo H, Choker G, Lenglet Y, Touret Y, Bouveret A, Grivard P, Le Roux K, Blanc S, Schuffenecker I, Courderec T, Arenzana-Seisdedos F, Lecuit M. Multidisciplinary prospective study of mother-to-child chikungunya virus infections on the Island of La Re 'union. PLoS Med. 2008;5(3): e60. doi:10. 1371/journal.pmed. 0050060

13. Seetharam KA, Sridevi K, Vidyasagar P. Cutaneous Manifestations of Chikungunya Fever. Indian Pediatric. 2012 jan; 49(1): 51-3. Epub 2011 Ago 15. PII: S097475591100033-2

14. Economopoulou A, Dominguez M, Helynck B, Sissoko D, Wichmann O. Atypical Chikungunya virus infections: clinical manifestations, mortality and risk factors for the severe disease during the 2005-2006 outbreak on Reunion. Epidemiol Infect. 2009;137: 534-41. 
15. Letters to the Editor. Vesiculobullous viral exanthem due to chikungunya in an infant Indian Dermatology Online Journal. 2014 ;5: Supplement Issue 2

16. Mittal A. Optic neuritis associated with chikungunya virus infection in South India. Optic neuritis associated with chikungunya virus infection in South India. Arch Ophthalmol. 2007 Oct;125(10): 1381-6.

17. Robin S, Ramful D, Le Seach F, Jaffar-Bandjee M-C, Rigou G, Alessandri J-L. Neurologic manifestations of pediatric chikungunya infection. J Child Neurol. 2008 Sep;23(9):1028-35. doi: 10.1177/0883073808314151. Epub 2008 Feb 20

18. Barr K.L, Vaidhyanathan V. Chikungunya in Infants and Children: Is Pathogenesis Increasing? Viruses. 2019;11: 294. doi:10.3390/ v1 1030294
19. Mittal A. Optic neuritis associated with chikungunya virus infection in South India. Optic neuritis associated with chikungunya virus infection in South India. Arch Ophthalmol. 2007 Oct;125(10): 1381-6.

20. H H Chua, MRCP. A Fatal Case of Chikungunya Virus Infection with Liver Involvement. J Malaysia. March 2010;65(1): 83-4.

21. Ritz N, Hufnagel M, Gérardin P. Chikungunya in Children. Pediatric Infectious Disease Journal. July 2015;34(7). Disponible en www.pidj.coml

22. Fernandes AIV, Souza JR, Silva AR, Cruz SBSC, Castellano LRC. Immunoglobulin Therapy in a Patient With Severe Chikungunya Fever and Vesiculobullous Lesions. Front. Immunol. 2019;10: 1498. doi: 10.3389/fimmu.2019.01498 